\title{
Synthesis of $\mathrm{Sb}_{2} \mathrm{~S}_{3}$ via Mechanochemical Milling and Its Application to Anode Material for Li-ion Battery
}

\author{
Chang-Yong Park1), Hyukjae Lee2)
}

\begin{abstract}
Antimony sulfide has a significantly higher theoretical capacity $(946 \mathrm{mAh} / \mathrm{g})$ as an anode material for Li-ion batteries than that of the widely used graphite $(372 \mathrm{mAh} / \mathrm{g})$, because antimony sulfide goes through a two-step electrochemical reaction with $\mathrm{Li}$ ions to complete a charge. In this study, the material and electrochemical properties of bulk antimony sulfide synthesized via dry vibration milling are investigated and compared with those of other commercially available antimony sulfide powders. We found that the synthesized antimony sulfide shows a finer and more spherical morphology than commercial antimony sulfide powders, which gives it higher charge-discharge capacities than the commercial antimony sulfide powders have. In particular, the cyclability of the synthesized antimony sulfide is much better than that of the commercial antimony sulfide when FEC additive is added to the electrolyte. The lithiation capacity at 50th cycle is $\sim 450 \mathrm{mAh} / \mathrm{g}$,
\end{abstract}

Keywords : Antimony Sulfide, Anode Materials, Capacity, Cyclability, Li-ion Battery

\section{Introduction}

Ever since its introduction in 1991, the Li-ion battery has been at center stage throughout the advance of small electronic devices because of its high energy density and low self-discharge. Recently, the Li-ion battery has broadened its application to large-scale energy storage devices, such as electronic cars[1]. Nevertheless, the basic material chemistry of Li-ion batteries has not changed much, especially the anode material. Carbon-based material has been a major anode material since the inception of Li-ion batteries. Although various types of carbon show excellent properties for secondary batteries, their capacity is limited to $372 \mathrm{mAh} / \mathrm{g}$. As more and larger devices adapt Li-ion batteries as their power sources, new anode materials with high capacity are required to meet this demand[2].

Received(June 29, 2018), Review Result(1st: July 9, 2018, 2nd: August 10, 2018), Accepted(September 10, 2018)

1) (Student) 36792 School of Materials Science \& Engineering, Andong National University, Andong, Korea email: ckddyd135@naver.com

2) (Professor, Corresponding Author) 36792 School of Materials Science \& Engineering, Andong National University, Andong, Korea

email: hlee@anu.ac.kr 
Antimony sulfide is one of the various anode candidates and has been investigated for Li-ion and Na-ion batteries. Although it has shown very high Li-ion storage capacity, a specific coating of nano-size powder has been required to achieve the desired capacity and cyclability [3-8], which would be a hurdle for commercialization because of the complexity in manufacturing and high cost. Hence, the primary goal of this study is to synthesize antimony sulfide by a simple route and evaluate whether it can be used as an anode material for Li-ion batteries. For this, antimony sulfide powder was synthesized using mechanochemical milling and its Li-electrochemical behavior was measured. For comparison, commercially available antimony sulfide powders were purchased and tested as well.

\section{Experiment}

The $\mathrm{Sb}_{2} \mathrm{~S}_{3}$ powder was synthesized via mechanochemical dry milling as follows. Sb (Aldrich, > $99.5 \%$ ) and S (Daejung, > 99.0\%) powders in the molar ratio of 2:3 were placed in a zirconia vial with a capacity of $45 \mathrm{~mL}$. Zirconia balls (diameter: $4 \mathrm{~mm}$ ) were also placed in the vial with the ball-to-powder ratio of 10:1. The high-energy dry milling was done with a vibratory mill (Spex SamplePro, 8000M), under Ar for 36h. Two commercially available reagent-grade $\mathrm{Sb}_{2} \mathrm{~S}_{3}$ powders, which will be denoted as reagent $\# 1$ and reagent $\# 2$, were purchased and used to make coin cells for comparison. $\mathrm{Sb}_{2} \mathrm{~S}_{3}$ powders were characterized by X-ray diffraction (XRD, Rigaku, D-Max2000), particle size analyzer (Mastersizer 2000), and scanning electron microscopy (Tescan, MYRA 3XM)

Electrochemical measurements were carried out using a coin-type half cell (CR2032), which was constructed in an Ar-filled glove box. The slurry for the working electrode consisted of 70 $\mathrm{wt} \% \mathrm{Sb}_{2} \mathrm{~S}_{3}$ as the active material, $10 \mathrm{wt} \%$ Super $\mathrm{P}$ carbon black as the conductor, and polyvinylidene (PVDF) in an N-methyl-2-pyrrolidone (NMP) solvent as the binder. The slurry was coated on a copper foil substrate using a Doctor Blade, followed by pressing and drying in vacuum at $120^{\circ} \mathrm{C}$ for $12 \mathrm{~h}$. Then $1.3 \mathrm{M} \mathrm{LiPF}_{6}$ in a mixture of ethylene carbonate and dimethyl carbonate (EC/DMC, 3:7 v/v) with $5 \mathrm{wt} \%$ of fluoroethylene carbonate (FEC) additive, Celgard 2400, and $\mathrm{Li}$ metal foil were used as the electrolyte, the separator, and the counter electrode, respectively. The cells were galvanostatically charged and discharged between $0.0 \mathrm{~V}$ and $2.0 \mathrm{~V}$ (vs. Li+/Li) at a current density of $100 \mathrm{~mA} / \mathrm{g}$, using a battery cycler (WonATech, WBCS3000). To investigate rate capability, the current density was raised to $250,500,1000$, and $2000 \mathrm{~mA} / \mathrm{g}$, and then dropped to $100 \mathrm{~mA} / \mathrm{g}$. 


\section{Results and Discussion}

Fig. 1(a) shows XRD patterns of the mechanochemically synthesized $\mathrm{Sb}_{2} \mathrm{~S}_{3}$ powder (MC syn 36 h) as well as two commercial powders (reagent \#1 and reagent \#2). Both commercial powders had well-defined peaks that corresponded to pure $\mathrm{Sb}_{2} \mathrm{~S}_{3}$. The mechanochemically synthesized powder showed weaker peak intensities over 0 to $80^{\circ}$, suggesting less crystallization; however, most peak positions were well matched to the pure $\mathrm{Sb}_{2} \mathrm{~S}_{3}$ phase. Particle-size distribution of each powder is shown in Fig. 1(b). Because of the high-energy milling of the starting powder, the synthesized $\mathrm{Sb}_{2} \mathrm{~S}_{3}$ showed smaller particles, especially less than the 1 Om range, than the commercial $\mathrm{Sb}_{2} \mathrm{~S}_{3}$ powders. Volume-weighted mean diameter of the synthesized $\mathrm{Sb}_{2} \mathrm{~S}_{3}$, reagent $\# 1$, and reagent \#2 were measured as $11.4,20.2$, and $23.2 \mu \mathrm{m}$, respectively.

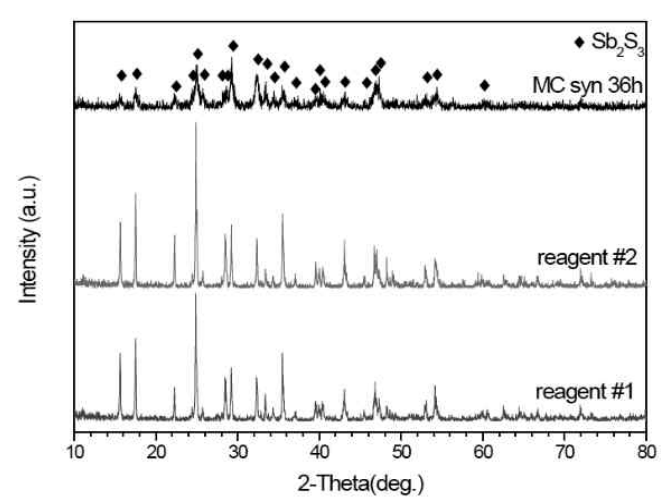

(a)

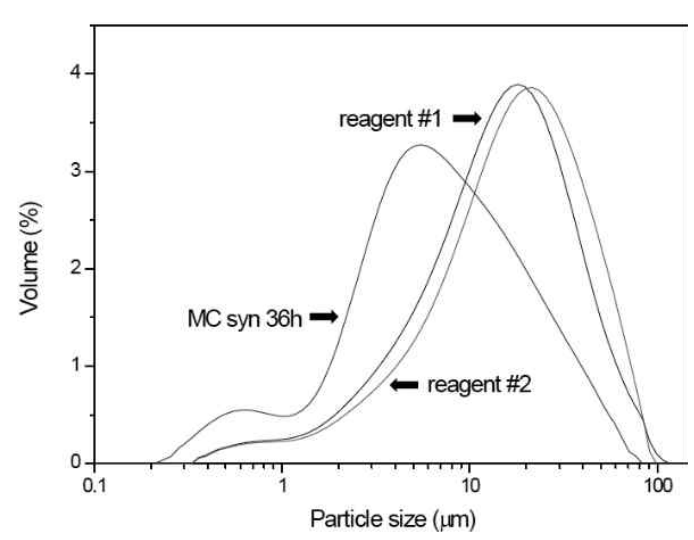

(b)

[Fig. 1] (a) XRD Patterns of $\mathrm{Sb}_{2} \mathrm{~S}_{3}$ Powders (b) Size Distribution of $\mathrm{Sb}_{2} \mathrm{~S}_{3}$ Powders

In general, smaller particles can improve the performance of an electrode by virtue of shortened Li-ion and electron travel paths inside the particle, so the better Li electrochemical behavior of a synthesized $\mathrm{Sb}_{2} \mathrm{~S}_{3}$ electrode is expected from the smaller particle-size distribution.

The morphologies of the $\mathrm{Sb}_{2} \mathrm{~S}_{3}$ powders are shown in Fig. 2. In the SEM images, the particles of the commercial powders have plate and rod shapes, whereas those of the synthesized $\mathrm{Sb}_{2} \mathrm{~S}_{3}$ powder are more spherical. Considering that the spherical shape compacts the powders more during the slurry coating, the spherical particles of the synthesized powder would work better for making an electrode. 

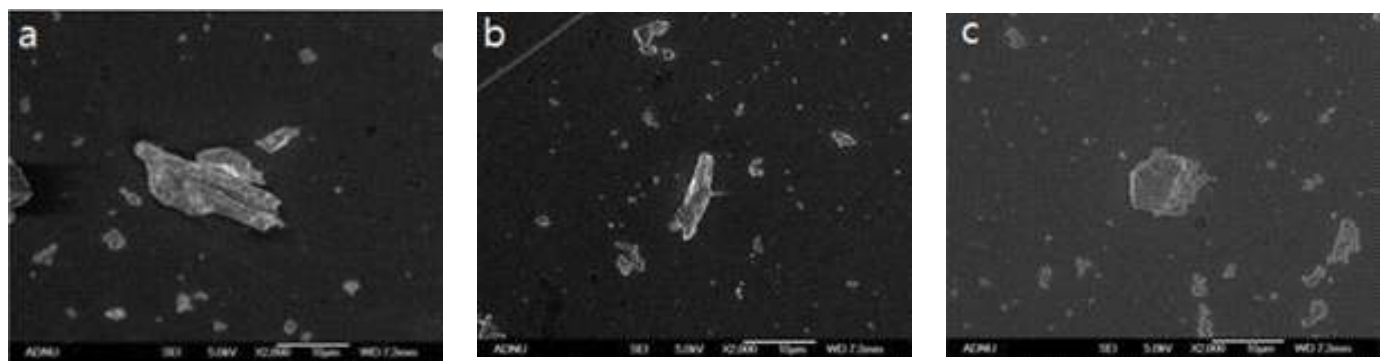

[Fig. 2] SEM Images of $\mathrm{Sb}_{2} \mathrm{~S}_{3}$ Powders (a) Reagent \#1 (b) Reagent \#2 (c) MC syn 36h

The charge-discharge profiles of $\mathrm{Sb}_{2} \mathrm{~S}_{3}$ electrodes at the current density of $100 \mathrm{mAh} / \mathrm{g}$ are shown in Fig. 3, where the profiles of the first, second, fifth, and tenth cycles are shown. When $\mathrm{Sb}_{2} \mathrm{~S}_{3}$ reacts with a $\mathrm{Li}$ ion, it can go through a two-step reaction:

$$
\begin{array}{ll}
\text { Conversion reaction: } & \mathrm{Sb}_{2} \mathrm{~S}_{3}+6 \mathrm{Li}^{+}+6 \mathrm{e}^{-} \leftrightarrows 2 \mathrm{Sb}+3 \mathrm{Li}_{2} \mathrm{~S} \\
\text { Alloying reaction: } & 2 \mathrm{Sb}+6 \mathrm{Li}^{+}+6 \mathrm{e}^{-} \leftrightarrows 2 \mathrm{Li}_{3} \mathrm{Sb} \\
\text { Net reaction: } & \mathrm{Sb}_{2} \mathrm{~S}_{3}+12 \mathrm{Li}^{+} 12 \mathrm{e}^{-} \leftrightarrows 3 \mathrm{Li}_{2} \mathrm{~S}+2 \mathrm{Li}_{3} \mathrm{Sb}
\end{array}
$$

The theoretical capacity of those two reactions is $946 \mathrm{mAh} / \mathrm{g}$, which is about 2.5 times larger than that of the most widely used anode, graphite $(372 \mathrm{mAh} / \mathrm{g})$. At the first cycle, the lithiation capacity of $\mathrm{Sb}_{2} \mathrm{~S}_{3}$ reaches $\sim 1000 \mathrm{mAh} / \mathrm{g}$, indicating that both the conversion and the alloying reaction proceeds to achieve the theoretical capacity. In the first lithiation profile, there are two plateaus at $\sim 1.4 \mathrm{~V}$ and $0.8 \mathrm{~V}$, which correspond to the conversion and alloying reactions, respectively. However, the capacity for the first delithiation is only 480 to $500 \mathrm{mAh} / \mathrm{g}$, and the second lithiation capacity is in the range of 440 to $490 \mathrm{mAh} / \mathrm{g}$, only about half of the first lithiation capacity. Since the plateau at $\sim 1.4 \mathrm{~V}$ (shown in the first lithiation profile) is missing in the first delithiation and the second lithiation profiles, the reverse reaction of (1) does not occur at the first delithiation, so that the $\mathrm{Sb}$ and Li2S, from the reverse reaction (2), are the final products of the first cycle. Hence, from the second cycle, the conversion reaction cannot contribute to the electrode capacity anymore. The inability to proceed in the reverse reaction of (2) is evident in the differential capacity plot, as shown in Fig. 4, in which the first, second, fifth, and tenth cycles are presented. For all three electrodes, the reaction peak at $1.4 \mathrm{~V}$, which can be seen in the first lithiation, vanishes completely from the second lithiation process. There is no significant change in reaction peaks corresponding to the alloying reaction, i.e., at $0.8 \mathrm{~V}$ during the lithiation, and at $1.05 \mathrm{~V}$ and $1.25 \mathrm{~V}$ during delithiation, with cycling, but the synthesized $\mathrm{Sb}_{2} \mathrm{~S}_{3}$ electrode is stabler in terms of peak position with cycling. 

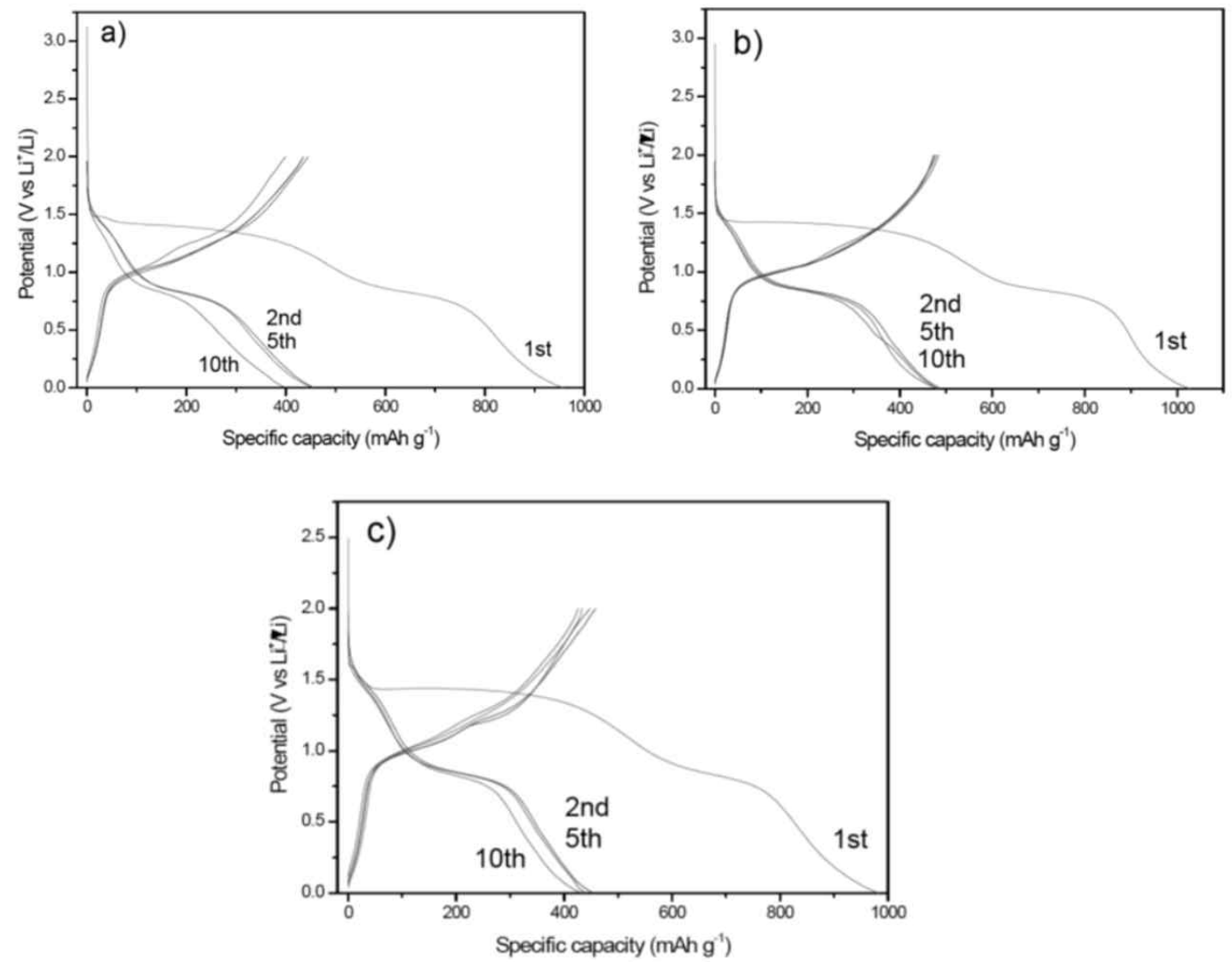

[Fig. 3] Charge-Discharge Profiles of (a) Reagent \#1 (b) Reagent \#2 (c) MC syn 36h

The cycle performances of the three $\mathrm{Sb}_{2} \mathrm{~S}_{3}$ electrodes at a current density of $100 \mathrm{~mA} / \mathrm{g}$ are shown in Fig. 5a. Because of the irreversible conversion reaction at the first lithiation, there are huge drops between the first and second lithiation capacities. The capacity retention of the second cycle is 44 to $47 \%$ of the capacity of the first cycle. At the early stage of cycling, all three electrodes show similar capacities, ca. $480 \mathrm{mAh} / \mathrm{g}$. As cycling goes on, the capacities of $\mathrm{Sb}_{2} \mathrm{~S}_{3}$ electrodes made of commercial powders keep decreasing with cycling, being less than 300 $\mathrm{mAh} / \mathrm{g}$ after 50 cycles. In contrast, the synthesized $\mathrm{Sb}_{2} \mathrm{~S}_{3}$ electrode shows very stable capacity with cycling, about $480 \mathrm{mAh} / \mathrm{g}$ after 50 cycles, displaying much better cycle performance than do the commercial $\mathrm{Sb}_{2} \mathrm{~S}_{3}$ powders tested in this study.

Normally, severe volume change is accompanied by alloying of $\mathrm{Li}$ with $\mathrm{Sb}$. The repeated volume changes during cycling cause the pulverizing and electrical disconnection of the electrode, leading to the capacity fade with cycling; i.e., the capacity retention is 44 to $55 \%$ of the capacity of the second cycle. Since the commercial powders' particles have plate- or 
rod-like shapes, these might be more vulnerable to the pulverizing caused by volume change. Since the synthesized $\mathrm{Sb}_{2} \mathrm{~S}_{3}$ powder's particles are small spheres, it can keep the integrity of particles even after prolonged cycling. Thus, the synthesized $\mathrm{Sb}_{2} \mathrm{~S}_{3}$ electrode shows about $455 \mathrm{mAh} / \mathrm{g}$ over 50 cycles, which is actually a little bit higher than the capacity of the second cycle, $\sim 432 \mathrm{mAh} / \mathrm{g}$.
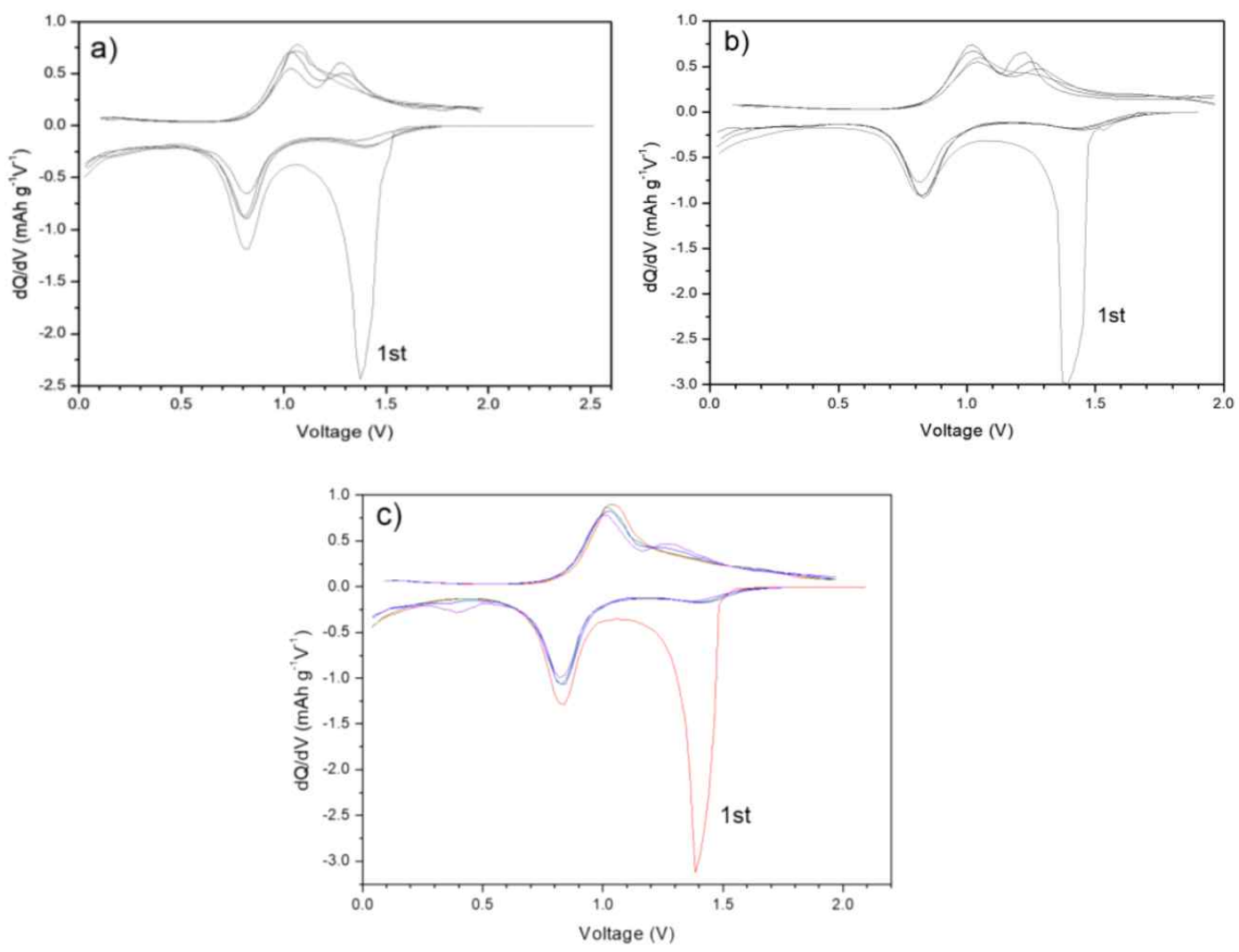

[Fig. 4] Differential Plots of (a) Reagent \#1 (b) Reagent \#2 (c) MC syn 36h

To investigate the rate capability of the $\mathrm{Sb}_{2} \mathrm{~S}_{3}$ electrode, the cells were cycled with different current densities; the results are shown in Fig. 5(b). At high current density, the particle size becomes a very critical factor to keep high capacity, because the travel path length of Li ions should be as short as possible. In this regard, the better rate performance of the synthesized $\mathrm{Sb}_{2} \mathrm{~S}_{3}$ powder in Fig. $5(\mathrm{~b})$ is not surprising, considering the smaller particle size of the synthesized $\mathrm{Sb}_{2} \mathrm{~S}_{3}$ powder. Note that the capacity of the $\mathrm{Sb}_{2} \mathrm{~S}_{3}$ electrode recovers to the same level when the current density is lowered, to $100 \mathrm{~mA} / \mathrm{g}$, after applying very high current density, of $2000 \mathrm{~mA} / \mathrm{g}$, in Fig. 5(b). 

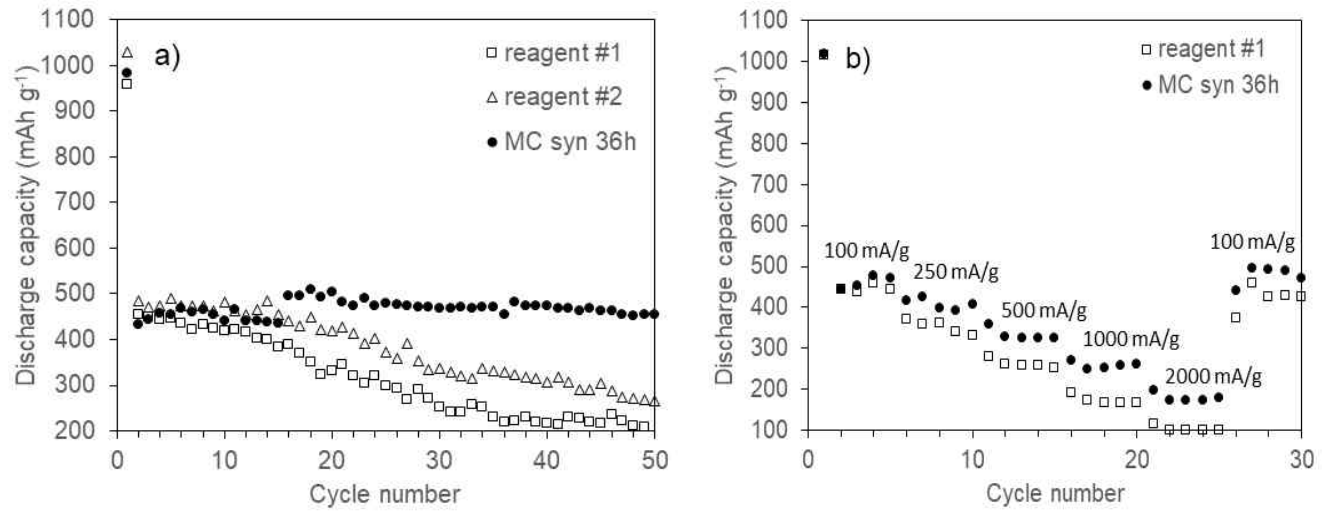

[Fig. 5] Lithiation Capacities of $\mathrm{Sb}_{2} \mathrm{~S}_{3}$ Electrode (a) $\mathrm{I}=100 \mathrm{~mA} / \mathrm{g}$, (b) Various Current Densities

\section{Conclusion}

Bulk $\mathrm{Sb}_{2} \mathrm{~S}_{3}$ powder was prepared using mechanochemical milling for anode material in Li-ion batteries. When used as a working electrode in a Li-ion coin cell, it showed better cyclability and rate capability than did the commercially available $\mathrm{Sb}_{2} \mathrm{~S}_{3}$ powders. However, the lithiation capacity over 50 cycles is only $\sim 450 \mathrm{mAh} / \mathrm{g}$, about half the theoretical capacity, $946 \mathrm{mAh} / \mathrm{g}$, because of the irreversible conversion reaction.

\section{Acknowledgement}

This work was supported by a grant from 2016 Research Funds of Andong National University

\section{References}

[1] G. E. Blomgren, The Development and Future of Lithium Ion Batteries, J. Electrochem. Soc., (2017), Vol.64, No.1, pp.A5019-A5025.

[2] D. Larcher and J-M. Tarascon, Towards greener and more sustainable batteries for electrical energy storage, Nature Chemistry, (2015), Vol.7, No.1, pp.19-29.

[3] A. Darwiche, C. Marino, M. T. Sougrati, B. Fraisse, L. Stievano, and L. Monconduit, Better cycling 
performances of bulk $\mathrm{Sb}$ in Na-ion batteries compared to Li-ion systems: an unexpected electrochemical mechanism, J. Am. Chem. Soc., (2012), Vol.134, No.51, pp.20805-20811.

[4] Y. Dong, S. Yang, Z. Zhang. J. M.. Lee, and J. A. Zapien, Enhanced electrochemical performance of lithium ion batteries using $\mathrm{Sb}_{2} \mathrm{~S}_{3}$ nanorods wrapped in graphene nanosheets as anode materials, Nanoscale, (2018), Vol.10, No.7, pp.3159-3165.

[5] A. S. Hameed, M. V. Reddy, J. L. T. Chen, B. V. R. Chowdari, and J. J. Vittal, RGO/Stibnite Nanocomposite as A Dual Anode for Lithium and Sodium Ion Batteries, ACS Sustainable Chem. Eng., (2016), Vol.4, No.5, pp.2479-2486.

[6] J. Kong, H. Wei, D. Xia, and P. Yu, High-performance $\mathrm{Sb}_{2} \mathrm{~S}_{3} / \mathrm{Sb}$ anode materials for Li-ion batteries, Mater. Lett., (2016), Vol.179, pp.114-117.

[7] Q. Ru, X. Chen, B. Wang, Q. Guo, Z. Wang, X. Hou, and S. Hu, Biological carbon skeleton of lotus-pollen surrounded by rod-like $\mathrm{Sb}_{2} \mathrm{~S}_{3}$ as anode material in lithium ion battery, Mater. Lett., (2017), Vol.198, pp.57-60.

[8] X. Zhou, L. Bai, J. Yan, S. He, and Z. Lei, Solvothermal synthesis of $\mathrm{Sb}_{2} \mathrm{~S}_{3} / \mathrm{C}$ composite nanorods with excellent Li-storage performanceElectrochem, Acta, (2013), Vol.108, pp.17-21. 